\title{
Reducing the iron burden and improving survival in transfusion-dependent thalassemia patients: current perspectives
}

This article was published in the following Dove Press journal:

Journal of Blood Medicine

8 August 2016

Number of times this article has been viewed

\section{Karim Bayanzay \\ Lama Alzoebie}

Department of Hematology, Gulf Medical University, Ajman, United Arab Emirates
Correspondence: Karim Bayanzay Gulf Medical University, PO Box 4I84, Ajman, United Arab Emirates Email KBayanzay@gmail.com

\begin{abstract}
Hypertransfusion regimens for thalassemic patients revolutionized the management of severe thalassemia; transforming a disease which previously led to early infant death into a chronic condition. The devastating effect of the accrued iron from chronic blood transfusions necessitates a more finely tuned approach to limit the complications of the disease, as well as its treatment. A comprehensive approach including carefully tailored transfusion protocol, continuous monitoring and assessment of total body iron levels, and iron chelation are currently the mainstay in treating iron overload. There are also indications for ancillary treatments, such as splenectomy and fetal hemoglobin induction. The main cause of death in iron overload continues to be related to cardiac complications. However, since the widespread use of iron chelation started in the 1970s, there has been a general improvement in survival in these patients.
\end{abstract}

Keywords: hematology, chelators, deferoxamine, deferiserox, deferiprone, liver iron concentration, iron overload, serum ferritin concentration, hepatic iron storage, iron chelation therapy

\section{Introduction}

The introduction of hypertransfusion regimens in the 1960s for thalassemic patients revolutionized the management of severe thalassemias; transforming a disease which previously led to early infant death into a chronic condition. Although chronic blood transfusion regimens have added decades to the lives of patients, clinicians are now faced with increasingly complicated management challenges. The devastating effect of the accrued iron from chronic blood transfusions necessitates a more finely tuned approach to limit the complications of the disease, as well as its treatment. Survival in transfusion-dependent thalassemia patients can be improved with proper understanding of the pathophysiology of thalassemia and iron toxicity, comprehensive transfusion protocols, accurate measurements of total body iron, and employment of strategies to reduce iron burden.

\section{Pathogenesis}

In the post-uterine life, two types of hemoglobins predominate, hemoglobin A1 and hemoglobin A2. Both of these are made up of four tetramers, either two $\alpha$ globulins and two $\beta$ globulins (hemoglobin A1) or two $\alpha$ globulins and two $\delta$ globulins (hemoglobin A2). In addition, a small percentage of fetal hemoglobin (HbF) is present and is made up of two $\alpha$ globulins and two $\gamma$ globulins. 


\section{$\beta$-Thalassemia}

The synthesis of $\alpha$ globulin and $\beta$ globulin is tightly regulated, such that the ratio is close to $1: 1$. In $\beta$ thalassemia there is a decrease or absence of the $\beta$ globulin leading to a relative increase in the number of $\alpha$ globulins. The excess and unpaired $\alpha$ globulins are less soluble and form precipitates that disrupt the normal function of red blood cells leading to a variety of clinical manifestations.

$\beta$-thalassemia is broadly clustered into three groups based on degree of clinical severity.

\section{$\beta$-thalassemia Minor}

$\beta$-thalassemia minor, also known as " $\beta$-thalassemia carrier" or " $\beta$-thalassemia trait", is the asymptomatic form of $\beta$-thalassemia. However, on rare occasions, some patients require blood transfusion during pregnancy due to severe anemia. Often, they are misdiagnosed as having iron deficiency, which is more common, until the diagnosis comes into question after a lack of response to iron supplementation. Once a carrier state is discovered, these patients should seek genetic counseling before having children.

\section{$\beta$-thalassemia Intermedia}

$\beta$-thalassemia intermedia usually presents in late childhood and requires infrequent to no transfusions. These patients still require proactive treatment to prevent serious complications, such as splenomegaly, extramedullary hematopoiesis, which may cause skeletal deformities and hypercoagulable states, which could lead to thrombotic events. Close monitoring, especially during periods of heightened stress, such as surgery, pregnancy, and rapid growth, is essential as this may exacerbate the condition to the extent that the implementation of a transient transfusion regimen may become necessary. Many clinicians advocate the use of early blood transfusion since it allows for normal growth and decreases the risk of developing extramedullary hematopoiesis. ${ }^{1}$ At the moment, no evidence-based guidelines have been developed for treatment of thalassemia intermedia and treatment is performed on an individualized basis, with the objective of limiting its long-term complications.

Interestingly, even without receiving regular blood transfusions, iron overload is still common among patients with thalassemia intermedia. The body compensates for the chronic state of anemia by increasing intestinal absorption of iron. Although the iron accumulates more slowly, it may eventually reach levels similar to those of transfusiondependent patients. Therefore, it is prudent to regularly monitor the iron levels and if elevated, especially in the liver and heart, iron chelation therapy should be initiated., ${ }^{2,3}$ Since the iron overload is more physiological, rather than iatrogenic, serum ferritin does not accurately reflect the true body iron. Magnetic resonance imaging (MRI) appears to be superior in determining iron levels in these patients. ${ }^{4}$

When patients with $\beta$-thalassemia intermedia reach old age, the accumulation of the adverse effects of decades of chronic anemia may lead to serious end organ damage such as cardiopulmonary compromise or signs of progressive bone marrow expansion (pathologic fractures, nerve impingement). To avoid these complications, initiation of chronic blood transfusion during this period may be necessary. The presence of non-transfusional iron overload makes the management of iron toxicity in these patients particularly challenging.

\section{$\beta$-thalassemia Major}

$\beta$-thalassemia major, also known as "Cooley's anemia" and "Mediterranean anemia" has a much earlier onset (6-24 months) and these patients require regular transfusions. Patients usually present with failure to thrive, progressive paleness, feeding problems, diarrhea, recurrent fever, or enlarged abdomen secondary to liver or spleen enlargement. Without treatment, the condition is further characterized by growth retardation, pallor, jaundice, poor musculature, genu valgum, hepatosplenomegaly, leg ulcers, development of masses from extramedullary hematopoiesis and skeletal changes. Mongoloid facies such as bossing, skull, depression of nasal bridge is also common. Since chronic blood transfusion protocols are applied primarily to patients with $\beta$-thalassemia major, the information presented in this review article will be primarily focused on patients with this subset of thalassemia.

\section{Alpha-thalassemias}

In $\alpha$-thalassemia, there is a decrease or absence in the production of $\alpha$ globulin. The severity of $\alpha$-thalassemia encompasses both ends of the spectrum. The most severe form, hemoglobin Barts, is incompatible with intrauterine life and the mildest forms, $\alpha$-thalassemia silent carrier and $\alpha$-thalassemia minor are essentially asymptomatic and do not require management. The only $\alpha$-thalassemia that may require chronic treatment is hemoglobin $\mathrm{H}$ disease. ${ }^{5}$ Although it is commonly classified under non-transfusion dependent thalassemias, ${ }^{6}$ these patients may end up requiring blood transfusions in the second decade of life. Regardless of chronic blood transfusions, they may still require iron chelation due to iron overload from increased intestinal iron absorption, analogous to $\beta$-thalassemia intermedia, and should be treated similarly. 


\section{Transfusion protocol}

The current practice guidelines for management of $\beta$-thalassemia major patients involve lifelong, regular blood transfusions. The primary goals of blood transfusion therapy include:

1. Maintaining optimal viability and function of the red blood cells to ensure sufficient oxygen transport to the tissues and organs to avoid the complications of chronic anemia and to suppress ineffective erythropoiesis.

2. Achieving an appropriate hemoglobin level while avoiding iron overload.

3. Avoiding adverse reactions of blood transfusion therapy with appropriate red cell antigen typing, cross matching, and screening for new antibody production. ${ }^{7}$

Chronic blood transfusions prevent most of the comorbidities of $\beta$-thalassemia major including growth, skeletal, and neurological complications. The decision to start chronic blood transfusion therapy requires input from the patient, family, and the medical team. Criteria for initiation of blood transfusion does not depend solely on the presence of anemia, but also on the inability to compensate for the anemia impacting the patient's quality of life and on the increasing symptoms of ineffective erythropoiesis.

Starting regular transfusions is indicated when initial hemoglobin level drops below $6 \mathrm{~g} / \mathrm{dL}$. However, anemia caused by sepsis or viral infection must be ruled out first by withholding transfusions and monitoring hemoglobin levels weekly. Thus, regular transfusions should be inaugurated with the goal to maintain the pre-transfusion hemoglobin levels $\geq 9 \mathrm{~g} / \mathrm{dL}$ in order to suppress extramedullary hematopoiesis, therefore minimizing skeletal changes, growth impairment, and splenomegaly.

The baseline laboratory investigations required before initiating blood transfusion include obtaining an extended red cell phenotype to reduce the risk of developing alloantibodies. In addition, antibodies to hepatitis B and C, and HIV should be acquired along with serum bilirubin, transaminase, and serum ferritin levels.

The blood product that is preferably administered is packed red blood cells depleted of leukocytes that are matched with the patient's red cell antigen phenotype with at least D, C, c, E, e, and Kell. Whole blood is unsuitable for chronic transfusions as the chance for non-hemolytic transfusion reaction to develop is common.

Blood transfusions are administered every 3 to 4 weeks at a rate of $15-20 \mathrm{~mL} / \mathrm{kg}$ to maintain the pre-transfusion hemoglobin level between 9 to $10 \mathrm{~g} / \mathrm{dL}$ and enough to suppress extramedullary hematopoiesis. Post-transfusion hemoglobin level should not exceed $14 \mathrm{~g} / \mathrm{dL}$. On the other hand, in patients with severe anemia with a hemoglobin level below $5 \mathrm{~g} / \mathrm{dL}$ or who have cardiac insufficiency, the rate of transfusion should be reduced to circumvent fluid overload, thus, diuretic usage may be necessary in some patients. Patients with cardiac compromise need to maintain a higher pre-transfusion hemoglobin level, between 10 to $12 \mathrm{~g} / \mathrm{dL} .{ }^{8}$

It is important that patients are monitored for the development of any hemolytic reactions developing during transfusion. Febrile and allergic reactions respond well to acetaminophen and diphenhydramine before administering future transfusions. Those that develop allergic reactions must be given washed packed red blood cell units.

Since iron overload is a critical issue of regular blood transfusions, it is very important to monitor the body's iron load. This includes obtaining monthly serum ferritin levels, monitoring growth charts in pediatric patients, looking for any signs of endocrinopathy, annual T2* MRI done from the age of 7 years, and an annual electrocardiogram to detect any arrhythmias in adolescents. To rule out any adverse effects of iron chelators an audiogram and a vision test is done yearly from the ages of 5 and 10 years respectively.

\section{Assessment of body iron}

Since $\beta$-thalassemia major patients require frequent blood transfusions to overcome the effects of anemia, iron overload is a common life-threatening complication. Therefore, it is crucial that reliable methods are available to monitor the iron status in transfusion-dependent patients.

Today, several techniques are available for evaluating iron overload, but some are more reliable than others. These include serum markers such as serum iron, ferritin, and total iron binding capacity. However, studies have found that serum ferritin values correlate poorly with hepatic iron concentration $^{9}$ and may give a false estimate of the total body iron. Also, serum ferritin may be influenced by a number of other factors, such as inflammatory states ${ }^{10}$ and hepatitis, which may show abnormally high serum ferritin levels, and vitamin $\mathrm{C}$ deficiency, which may show abnormally low serum ferritin levels. ${ }^{9}$ In addition, serum ferritin is not useful in predicting iron overload-related complications. Despite some patients having low ferritin levels for decades, they may still experience heart diseases as they age. ${ }^{11}$ On the other hand, serial serum ferritin values are useful in monitoring iron chelator responsiveness as this method is easy, cheap, and easily accessible. ${ }^{10}$

Since serum ferritin levels underestimate the body's total iron, some centers select more invasive procedures to assess iron overload in transfusion-dependent patients. Since the liver is the predominant iron storage organ, the liver iron 
content (LIC) correlates well with the body's total iron status. Unfortunately, determination of the LIC requires a liver biopsy, which is more expensive, invasive, and carries serious risks, such as hemorrhage. ${ }^{10}$

To overcome the drawbacks of liver biopsy, several non-invasive techniques have arisen to estimate tissue iron concentration. These include the superconducting quantum interference device (SQUID), quantitative computed tomography $(\mathrm{CT})$, and $\mathrm{MRI} .^{10}$

Among the first non invasive techniques used to measure tissue's iron load was SQUID. ${ }^{11}$ SQUID utilizes very low power magnetic fields with sensitive detectors placed over the liver to measure the magnetic flux created from the interference of the patient's body iron with the field. ${ }^{12}$ Although the test has shown linear correlations with LIC measured through liver biopsy, SQUID tends to underestimate LIC values. Additionally, its use is restricted due to limited availability and high costs. ${ }^{9}$

CT is a well-tolerated, and relatively inexpensive alternative for determining LIC. A non-contrast CT in patients with hepatic hemochromatosis shows a diffuse increase in liver attenuation (usually $>75$ Hounsfield units). Consequently, the liver vasculature appears more prominent because of the increased contrast between the vessels and the highly attenuated liver as shown in Figure 1.

Though CT provides a closer correlation with LIC than serum ferritin levels, ${ }^{13}$ this technique has been limited by poor sensitivity in patients with low iron levels and increased exposure to ionizing radiation. ${ }^{11}$

Finally, MRI is the most accurate and widely available tool in detecting and screening for iron overload in endocrine organs, the liver, and the heart. It is currently the preferred imaging modality because of its several advantages including a low rate of variability between measurements and a high sensitivity. ${ }^{10}$ It has been integrated in the standard of care in not only transfusion-dependent thalassemic patients, but in all chronic transfusion-dependent anemic patients.

To measure iron concentration in a particular tissue, the MRI transmits a stimulus that excites the water's protons in the tissue. As the protons relax back to their steady state, they send out microwaves to the MRI scanner which are interpreted into an image. The magnetic environment in non-iron overloaded tissues is homogenous, giving a brighter image and the signals last longer (Figure 2). On the other hand, in iron overloaded tissues, iron acts like a magnet disrupting the coherence between the protons and darkening the image quicker (Figure 3). ${ }^{10}$ This darkening process which is proportional to tissue iron concentration is described by a "half-life" as T2* with a gradient echo imaging and $\mathrm{T} 2$ which is provided by a spin echo. The rate of darkening is defined as $\mathrm{R} 2 *$, which is a reciprocal of $\mathrm{T} 2 *$. In summary, the greater the tissue iron load, the shorter the signal half-life, thus giving a smaller T2 and T2* on the MRI. ${ }^{11}$

Research is still underway to find more accurate and less invasive methods of measuring total body iron. A recent study has shown that total body iron can be measured and monitored by applying a Perl's Prussian blue stain to exfoliative buccal cells of $\beta$-thalassemic major patients. These results were considered significant, however, further research is required to support these findings. ${ }^{14}$

It is important that chronically transfused patients have serial follow-ups to determine whether the patient is responding to chelation therapy properly in order to avoid the lifethreatening complications of iron overload on the tissues, however, the technique needs to be easy, sensitive, cheap, and widely available. Thus, several institutes have adopted the MRI as an effective technique to serially monitor iron overload in chronic transfusion patients.
A

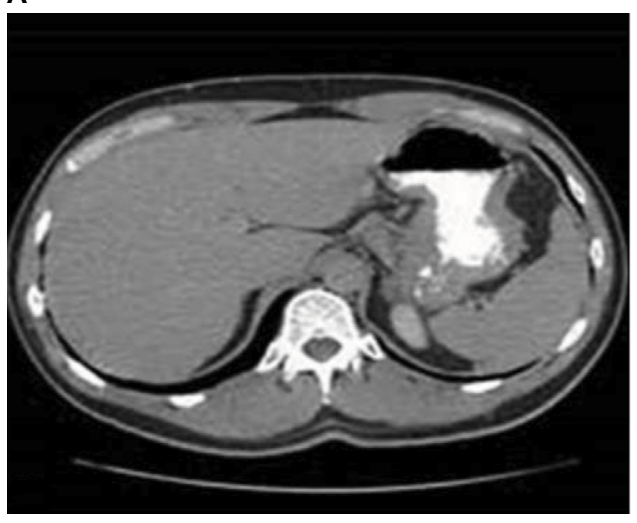

B

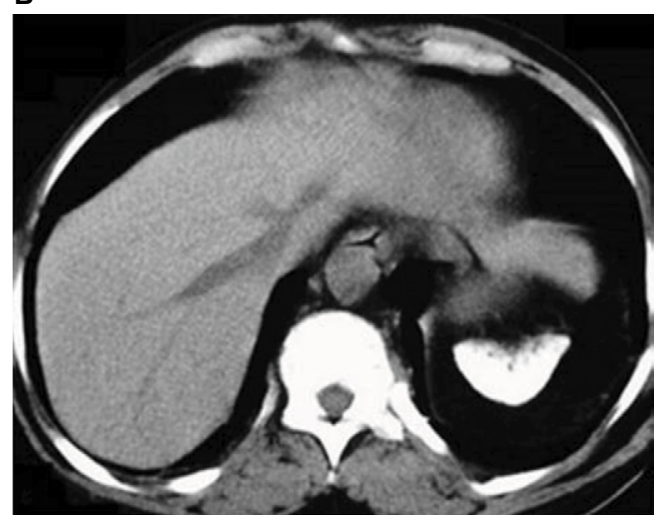

Figure I Non-contrast CT scans.

Notes: (A) Normal liver and spleen. (B) Diffuse increased attenuation of the liver indicating excess iron deposition in a 47 -year old patient with sickle cell disease undergoing multiple blood transfusions.

Abbreviation: CT, computed tomography. 


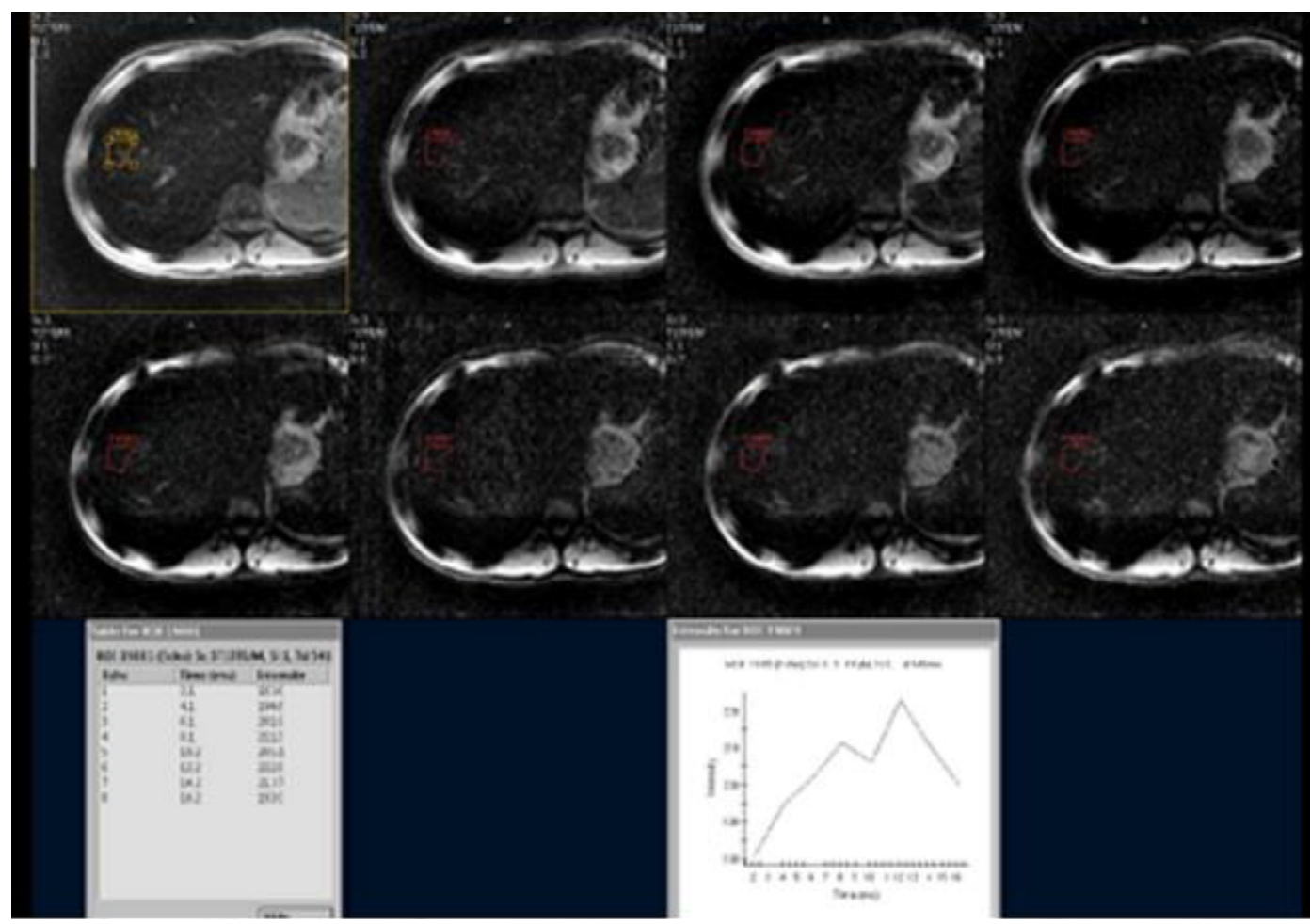

Figure 2 Normal axial T2* of liver of a 21 -year old male patient with a known case of $\beta$-thalassemia on regular blood transfusions. Notes: There is a rise in signal intensity as echo time increases. This patient had a normal liver iron concentration of $0.15 \mathrm{mg} / \mathrm{g}$.

A

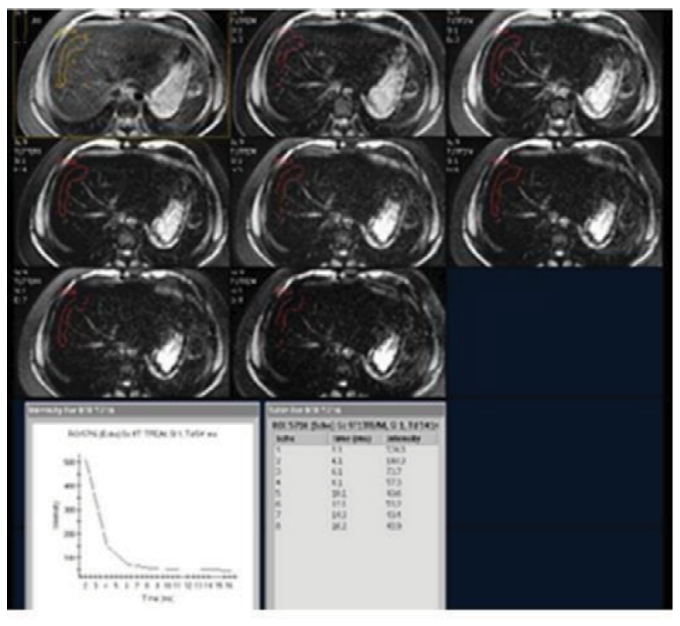

B

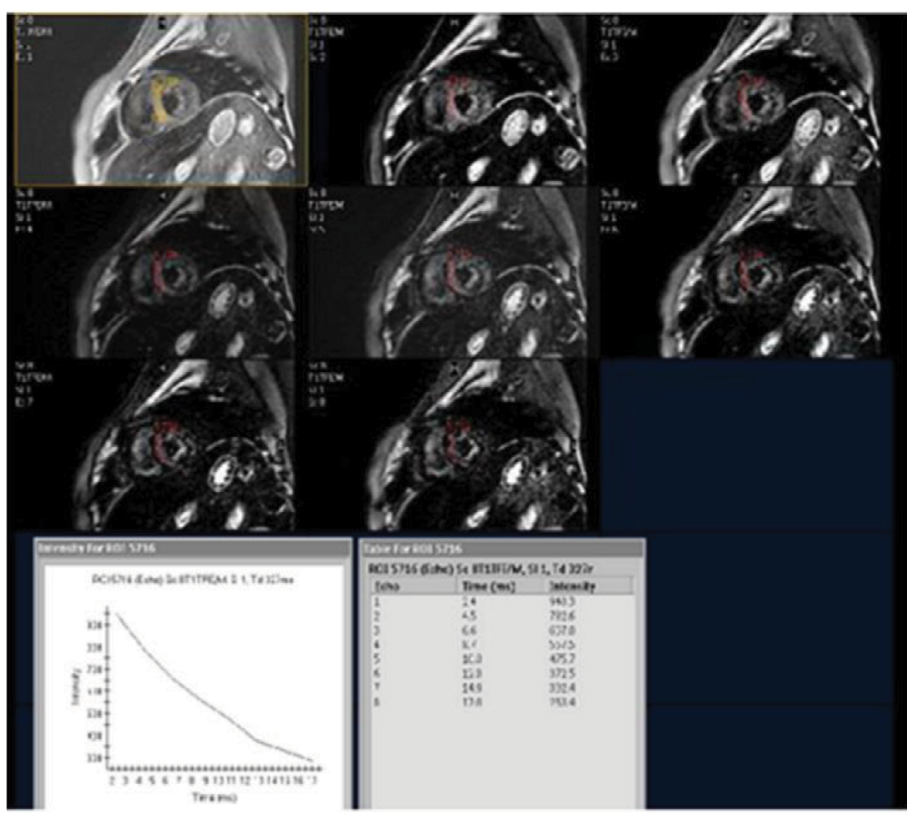

Figure 3 Axial T2* of liver and heart of a 24-year old female patient with a known case of $\beta$-thalassemia major on chronic blood transfusions.

Notes: (A) Moderate-to-high degree of hemosiderosis in this patient's liver. T2* measurements of liver demonstrated sever hepatic iron loading of 2.1 ms. Sharp decrease in signal intensity as the echo time increases. Measured liver iron content was moderately high with $13.09 \mathrm{mg} / \mathrm{g}$. (B) Heart - moderate degree of cardiac hemosiderosis demonstrating a gradual decrease in signal intensity as the echo time increases.

\section{Iron toxicity}

Each unit of transfused blood contains 200 to $250 \mathrm{mg}$ of elemental iron. The body has not developed adequate mechanisms to excrete such quantities of iron. Therefore, the development of iron overload in patients receiving chronic blood transfusion is inevitable. After the iron storage ability of the reticuloendothelial cells is saturated, the iron spills into blood where it normally binds to transferrin. Once transfer- 
rin is oversaturated the level of non-transferrin bound iron steadily increases. The unbound iron begins to accumulate in various organs, such as the heart, liver, pancreas, gonads, and pituitary, and causes the catalysis of injurious compounds, such as free radicals. These free radicals begin to damage cells leading to fibrosis or organ dysfunction. ${ }^{15}$

When the unbound iron is taken up by cardiac tissue, the steady accumulation of oxygen metabolites and other toxin formed from reactions catalyzed by the iron ultimately destroy the tissue. Eventually, there is development of left ventricular hypertrophy and conduction disturbances in late childhood. If untreated, these patients may develop refractory congestive heart failure by their mid-teens. ${ }^{16}$

Histological studies have shown deposition of iron in pituitary gland leading to hypogonadotropic hypogonadism and growth hormone deficiency, despite iron chelation therapy. ${ }^{17-19}$

\section{Reducing iron burden}

Currently the mainstay of managing transfusion-related iron toxicity is through the use of iron chelators, however, other strategies to limit iron burden are also employed.

\section{Iron chelators}

Iron chelators work by neutralizing unbound iron and removing excess iron in tissues. Iron has six active sites and to achieve complete inactivation of a single atom of iron, all six sites must be bound by the chelator. Hexidentate chelators, such as deferoxamine, can bind to all six sites, while tridentate chelators such as deferasirox can bind to three sites and bidentate chelators, such as deferiprone, can bind to only two sites. Therefore, a hexidentate chelator binds at a 1:1 ratio, while a tridentate chelator binds at a 2:1 ratio, and a bidentate chelator binds at a $3: 1$ ratio. The non-hexidentate chelators, deferasirox and deferiprone, will produce a mixture of irons with variable levels of inactive sites and only a minority of those irons will be completely inactive.

The use of iron chelating agents has significantly increased the survival rates and decreased morbidity related to organ system toxicity. Ample numbers of studies have illustrated that iron chelators are effective in ceasing and often reversing liver and cardiac dysfunction attributed to iron overload. ${ }^{16,20}$ Chelation therapy has also been shown to reduce the risk of diabetes mellitus and glucose intolerance ${ }^{21}$ and, if used early, allows most patients to reach normal puberty, and increases fertility rates. ${ }^{22}$

Currently, there are a variety of iron chelators, each with different properties, to choose from. An ideal chelator should bind to a large amount of ferric iron at low doses and toxicity, while having a low affinity for ferrous iron and other metals. Since iron often enters the cell during iron overload, intracellular penetration is also important. For better compliance, the drug must have good oral tolerability, affordability, and availability.

\section{Deferoxamine}

Deferoxamine is the first iron chelator to be used in treatment of iron toxicity. Since deferoxamine has been around for over 50 years, there has been significant clinical experience. Due to its established efficacy it continues to be the standard iron chelator used. In a 10-year New England Journal of Medicine study published in 1994, all pediatric patients who were able to achieve controlled serum ferritin levels with 12-hour subcutaneous deferoxamine infusion survived without cardiac disease. ${ }^{23}$ Other studies have confirmed this, showing a significant decline in mortality and heart failure. ${ }^{23,24}$

Deferoxamine is capable of reversing hepatic iron overload. In the liver, deferoxamine is internalized by hepatic parenchymal cells where it binds with intracellular iron and is excreted in bile. Studies in pediatric groups showed that intermittent deferoxamine infusion decreased iron storage in the liver and prevented hepatic fibrosis. ${ }^{20}$ However, patients with concurrent chronic hepatitis may require dose adjustments. ${ }^{25}$

Deferoxamine is also able to remove iron from cardiac cells. Iron released by macrophages is immediately chelated and excreted in urine. Within a few hours of administration, the urinary iron concentrations increase significantly in a dose-dependent fashion. ${ }^{26}$ Each nightly infusion should have a loss of 20 to $50 \mathrm{mg} /$ day of iron in urine and stool, which should be effective at controlling iron levels, if the patient is receiving under four units of packed red blood cells per month. $^{27}$

The disadvantages of deferoxamine are related to its short half-life and parenteral requirements. Patients usually require nightly continuous subcutaneous infusions 5-7 days/week of $2 \mathrm{~g}$ deferoxamine via a pump. This is not only difficult on the patient, but is labor intensive and necessitates the installation of semi-permanent intravenous access, which is associated with additional complications. ${ }^{28}$ Subcutaneous injections are an alternative, but are painful. Both avenues increase the likelihood of non-compliance with therapy, which may be as low as 50\% in children and adolescent populations. Patients who are not fully compliant with deferoxamine treatment have substantially greater morbidity and mortality as well as increased costs..$^{29}$ Therefore, many investigators have pushed for oral agents. 
Some reports have suggested that early and aggressive deferoxamine administration may adversely affect skeletal maturation and result in growth retardation. ${ }^{30}$ However, there is also evidence that early chelation may delay cardiac symptoms and prolong survival, therefore some centers choose to begin deferoxamine infusions around 5 years of age. ${ }^{31}$

Overall, deferoxamine is well tolerated by most patients. Many patients experience minor local irritation at the injection site, which can be controlled by reducing the dosage or the rate of infusion. Therefore, frequent monitoring for complications is important. These complications include: ${ }^{32}$

1. Ototoxicity, which is often irreversible, can occur when the ratio of deferoxamine $(\mathrm{mg} / \mathrm{kg})$ to serum ferritin $(\mu \mathrm{g} / \mathrm{L})$ is greater than $0.025 .{ }^{33}$ Therefore, audiology exam should be carried out before treatment. Another screening should be performed after 6 months and another formal audiogram should be administered 12 months into treatment. It is important to detect any hearing loss early because decreasing the dosage can prevent hearing loss. ${ }^{34}$

2. Ocular toxicity may present as night blindness, blurred vision and decreased visual acuity, impaired color vision or cataract. An ophthalmologist should evaluate children every 6 months and adults annually as well as routinely asking about vision changes during visits.

3. Retarded growth and skeletal changes.

4. Infections, such as Yersinia enterocolitica and other pathogens.

5. Pulmonary and neurological disease is associated with doses greater than $60 \mathrm{mg} / \mathrm{kg}$.

6. Renal function may be affected, therefore creatinine/ blood urea nitrogen should be monitored every 3 months. Decrease dosage if serum creatinine increases by $30 \%$ to $50 \%$ from baseline and discontinue temporarily if the serum creatinine rises by greater than $50 \%$ from baseline or if the protein/creatinine ratio is greater than $0.6 \mathrm{mg} / \mathrm{mg} .{ }^{35}$

7. Liver function should be monitored every 3 months and chelators should be stopped if ALT is greater than five times the upper limit.

Many serious complications can be avoided by utilizing doses less than $2.5 \mathrm{~g}$ with each infusion. Once serious complications develop, they can generally be controlled by discontinuing the drug or decreasing its dosage. These complications, coupled with poor compliance as a result of inconvenient parenteral administration, has limited the effectiveness of this treatment as a chelator. The need for a better oral iron chelator was readily apparent and so the development of these alternatives was undertaken.

\section{Deferiprone}

Deferiprone was introduced in the 1980s and was the first oral iron chelator to be used and works by forming stable complexes with plasma iron that can be excreted through urine. ${ }^{36}$ Clinical experience over the past decades have shown deferiprone to be effective in increasing iron excretion, however, deferoxamine still appears to be slightly more effective. This has been attributed to fecal iron excretion as a result of deferoxamine.$^{37}$ A 2006 randomized controlled trial concluded deferiprone to more effective in improving myocardial siderosis than deferoxamine. ${ }^{38}$ However, a follow-up metaanalysis in 2008 , failed to draw the same conclusion, rather, they found that there is no significant difference between deferoxamine and deferiprone in reducing iron concentration. ${ }^{39}$ For the moment, it seems more studies and longer follow-ups are required before a definitive conclusion can be reached.

Like deferoxamine, deferiprone has a short half-life which requires multiple daily dosing. ${ }^{40}$ The recommended dosage is between $75-100 \mathrm{mg} / \mathrm{kg} / \mathrm{day}$ divided into three doses. The most serious adverse effect of deferiprone is reversible agranulocytosis, usually within the first year, but reported up to 19 months. ${ }^{36,41}$ This effect tends to recur with continuation of treatment. At the moment, it is not clear whether this is an idiosyncratic effect or dose-related. Due to this reaction, weekly monitoring of white blood cells in the first year and then 2 weeks thereafter is recommended to detect early neutropenia or agranulocytosis. Discontinue if the neutrophil levels drop below 1,500 cell $/ \mu \mathrm{L}$.

Gastrointestinal tract symptoms, such as nausea, vomiting, and abdominal pain have also been reported in up to $33 \%$ of patients, however, they are typically mild..$^{42}$ Zinc deficiency is a rare occurrence, usually seen in diabetic patients. Hence, zinc levels should be checked every 3 to 6 months in diabetic patients. Provide zinc supplements if zinc is determined to be low.

Deferiprone may also lead to elevated liver enzymes, which may be attributed to other associated conditions such as hepatotoxicity. Chelation therapy should be halted if ALT is five times the upper limit of normal or if transaminases are persistently two times the upper limit of normal. ${ }^{43}$

Although compliance is substantially higher with deferiprone than deferoxamine, ${ }^{29}$ the efficacy and safety of deferiprone still have not been established, which has precluded deferiprone from routine use. ${ }^{44}$ Many researchers have begun to study whether a combination treatment of deferoxamine and deferiprone is more effective than monotherapy. A 2007 randomized, placebo-controlled, double-blind trial comparing combination versus monotherapy concluded combination 
therapy to be more beneficial in improving cardiac function and reducing cardiac iron burden.$^{45}$ However, a subsequent 2013 meta-analysis did not find enough evidence to warrant a recommendation to begin combination therapy in all patients. Their recommendation maintained that deferiprone should be reserved for patients in whom deferoxamine is contraindicated or inadequate. ${ }^{46}$

\section{Deferasirox}

Deferasirox is the latest oral iron chelator and has undergone extensive studies including a Phase 3 clinical trial. The trial indicated that daily administration of $30 \mathrm{mg} / \mathrm{kg} /$ day could decrease LIC similar to deferoxamine at $50 \mathrm{mg} / \mathrm{kg} /$ day. The long half-life of deferasirox (8-16 hours) allows for once-daily dosing, which is one of the major advantages of this drug. ${ }^{47}$

The CORDELIA study, was a prospective, randomized study that compared the usage of deferasirox versus deferoxamine for myocardial iron removal in $\beta$-thalassemic major patients with myocardial siderosis, but no cardiac malfunction. This study demonstrated that there is no superiority of deferoxamine compared to deferasirox for myocardial iron removal. ${ }^{48}$

During the trial, only mild adverse effects, such as abdominal pain, back pain, and skin rash were reported. However, now post-marketing reports of acute renal failure, cytopenias, hepatic failure, and gastrointestinal hemorrhage, often fatal, have surfaced. It has already been noted that these reactions were more frequent in patients with advanced age, high-risk myelodysplastic syndromes, underlying renal or hepatic impairment or low platelet counts. ${ }^{49}$ Many studies have also reported the presence of acquired proximal renal tubular dysfunction. ${ }^{50,51}$

Consequently, it is important to routinely perform liver function tests and renal function tests. It is generally advised to discontinue the chelator if serum creatinine is greater than two times normal or increases by $50 \%$ from baseline or if the protein-to-creatinine ratio is $>0.6 \mathrm{mg} / \mathrm{mg}$. It should also be discontinued if the serum ALT is greater than five times the upper limit.

\section{Choosing the chelator}

No iron chelating agent is considered "gold standard" and clinicians differ on the optimum approach to initiating iron chelation therapy in patients with transfusion-dependent thalassemia. Moderate doses of deferasirox can maintain iron balance in a majority of patients on blood transfusion. It tends to be preferred over deferoxamine because of greater compliance, especially since poor compliance is associated with poor survival. ${ }^{29}$ However, it must be explained to patients that more data on long-term efficacy have been established for deferoxamine.

The choice of specific iron chelator is based on factors other than the efficacy of the drug. Other factors such as age, patient preference, comorbidities, and drug side effects will ultimately determine which chelator is used (Table 1). Patients with high iron intake may require higher dose deferasirox or combination regimens with deferoxamine. Deferiprone remains as a second line drug and plays a larger role if there is significant cardiac toxicity from iron overload.

Table I Comparison of iron chelators

\begin{tabular}{|c|c|c|c|}
\hline Preperty & Deferoxamine & Deferiprone & Deferasirox \\
\hline Chelator: Fe & $\mathrm{I}: \mathrm{I}$ & $3: 1$ & $2: 1$ \\
\hline Route of administration & $\begin{array}{l}\text { Subcutaneous } \\
\text { Intravenous } \\
\text { Implantable delivery system }\end{array}$ & Oral & Oral \\
\hline Usual dosage & $25-50 \mathrm{mg} / \mathrm{kg} /$ day & $75-100 \mathrm{mg} / \mathrm{kg} /$ day & $20-30 \mathrm{mg} / \mathrm{kg} / \mathrm{day}$ \\
\hline Schedule & Administration overt 8-24 hours 5-7 days a week & $3 \times$ daily & Daily \\
\hline Half-life & Short (minutes) & Moderate (hours) & Long (8-46 hours) \\
\hline Adverse effects & $\begin{array}{l}\text { Local reactions } \\
\text { Retinal toxicity } \\
\text { Auditory toxicity } \\
\text { Pulmonary toxicity }\end{array}$ & $\begin{array}{l}\text { Agranulocytosis, with mild } \\
\text { neutropenia } \\
\text { Arthralgia/erosive arthritis }\end{array}$ & $\begin{array}{l}\text { Abdominal discomfort with rash } \\
\text { or mild diarrhea; mild elevation } \\
\text { in creatinine }\end{array}$ \\
\hline Monitoring & $\begin{array}{l}\text { Hearing test } \\
\text { Vision test } \\
\text { Growth assessment } \\
\text { Pulmonary function } \\
\text { Renal function } \\
\text { Liver Function }\end{array}$ & $\begin{array}{l}\text { CBC with differential Zinc } \\
\text { Liver function }\end{array}$ & $\begin{array}{l}\text { CBC with differential } \\
\text { Platelet count } \\
\text { Liver function } \\
\text { Renal function }\end{array}$ \\
\hline Advantages & Long-term data available & $\begin{array}{l}\text { May be superior in removal of } \\
\text { cardiac iron }\end{array}$ & Once daily dosage \\
\hline
\end{tabular}

Abbreviation: $\mathrm{CBC}$, complete blood count. 
In certain cases, monotherapy may be insufficient to achieve treatment goals. At this point the clinicians should either increase the current dose of the drug or switch to a different chelator. However, if treatment goals are still not achieved, then the clinician may opt to initiate combination therapy. Combination chelators tend to be more effective than monotherapy because chelators utilize different mechanisms for removing iron. Combination therapy has been shown to be useful for patients with mild-to-moderate hepatic and cardiac iron overload, as well as severe degrees of cardiac siderosis and impaired left ventricular function..$^{52,53}$

\section{When to start iron chelators}

Starting a vigorous iron chelation therapy earlier has been associated with significant improvement in survival. ${ }^{54}$ The general rule is to begin iron chelation treatment when the following parameters are present: ${ }^{55}$

1. After transfusion of $100 \mathrm{~mL} / \mathrm{kg}$ packed red blood cells (10-20 transfusions).

2. Ferritin levels above $1,000 \mathrm{ng} / \mathrm{mL}$.

3. Liver iron concentration greater than $3 \mathrm{mg} / \mathrm{g}$ dry weight of liver as measured by liver biopsy or MRI.

\section{Dosing}

No set dosing has been specified and most clinicians tailor dosing to achieve particular laboratory parameters. Dosing is steadily increased until serum ferritin levels are $<1,000 \mu \mathrm{g} / \mathrm{L}$, LIC $<7 \mathrm{mg} \mathrm{Fe} / \mathrm{g}$ dry weight, and cardiac T2* by MRI $>20$ millisecond. It can take anywhere from 3 to 5 years to reduce serum ferritin levels below $1,000 \mathrm{ng} / \mathrm{mL}$ with deferoxamine alone. Once serum ferritin falls below 300 to $500 \mu \mathrm{g} / \mathrm{L}$ and $/$ or LIC falls below $3 \mathrm{mg} / \mathrm{g}$ dry weight, chelation therapy can be reduced or discontinued..$^{45}$ Dosage should be increased if ferritin and LIC levels increase on average over a period of 6 months. Keep in mind not to exceed levels which may lead to complications, as discussed earlier.

Intensification of treatment with increased dosage, frequency or combination chelators should be considered when: LIC $>15 \mathrm{mg} \mathrm{Fe} / \mathrm{g}$, serum ferritin $>2,500$, cardiac T2* MRI $<15$ milliseconds or a fall in left ventricular ejection fraction due to siderosis, cardiac failure or arrhythmia. Often patients on deferoxamine are noncompliant and therefore these patients should be switched to deferiprone. ${ }^{52,53}$

\section{Improvements in survival}

Survival of patients with $\beta$-thalassemia major is influenced by several factors ranging from age, education, ferritin levels, complications, and many more. However, over the past few decades a steady increase in survival has been documented. This was highlighted in a 2005 study where survival probabilities estimated by the Kaplan-Meier method displayed that survival was higher for patients born after 1975 compared to those born before. ${ }^{56}$

Despite these advances the most common cause of death remains cardiac complications $(68 \%)$ due to iron overload. ${ }^{24}$ Therefore, control of iron overload remains the best prognostic factor. Despite the problems with using serum ferritin as a measure of total body iron, as mentioned earlier, results from several studies including those carried out in Iran and Italy, have suggested that serum ferritin levels affect the longevity of thalassemic patients. These studies emphasize that serum ferritin levels $>2,500 \mathrm{ng} / \mathrm{mL}$ are associated with reduced survival. ${ }^{24,57}$

A study carried out in 1991 divided patients into two groups. Group 1 followed a low-transfusion protocol with no chelation, whereas Group 2 followed a hypertransfusion regimen with deferoxamine. Group 1 had an estimated median age of survival of 17.4 years compared to 31 years in Group 2. This study concludes deferoxamine treatment, when used in amounts proportionate to the iron burden, can delay cardiac complications and prolong survival. ${ }^{58}$

\section{Splenectomy}

Splenectomies allow red blood cells to live longer and therefore the patient can maintain higher levels of hemoglobin for longer periods and decrease their requirement for transfusions. Splenectomies are performed when the transfusion requirements begin to exceed $200 \mathrm{~mL} / \mathrm{kg}$ or the transfusion requirements increase by $50 \%$ over a 1 -year period. Unfortunately, removal of the spleen does have other complications, such as increased risk of infection and thrombosis, therefore the benefits and risk of splenectomies must be carefully weighed before initiating this course of treatment. ${ }^{31}$ It is advisable to delay splenectomy until after the age of 5 years, when proper immunizations can be dispensed to reduce the increased risk of post-splenectomy infection. ${ }^{59}$

\section{Induction of $\mathbf{H b F}$}

As mentioned earlier, $\mathrm{HbF}$ does not have a $\beta$ globulin, therefore by inducing more $\mathrm{HbF}$, it may serve to correct the excess $\alpha$ chain imbalance. Normally, HbF makes up less than 3\% of the total hemoglobin, however, certain cytotoxic drugs and cancer chemotherapy agents are able to increase the production of $\mathrm{HbF}$, such as decitabine, butyrate, and hydroxyurea.

Many studies have shown a decrease in transfusion requirements or complete transfusion independence, after the 
administration of hydroxyurea. ${ }^{60,61}$ Therefore, $\mathrm{HbF}$ induction is an effective strategy to reduce iron burden by decreasing the frequency of transfusions. Unfortunately, hydroxyurea is unable to induce the production of $\mathrm{HbF}$ in all patients and treatment responses are highly variable. Currently, no randomized controlled study has been done, so more studies are required before stronger conclusions about the efficacy of hydroxyurea can be drawn.

Although no ideal HbF inducer exists, the therapeutic benefits of $\mathrm{HbF}$ induction is undeniable. Currently, many clinicians administer fetal globulin inducers on a trial basis, especially to patients with high transfusion requirements and patients experiencing complications from blood transfusions, such as alloimmunization.

\section{Conclusion}

Thalassemia syndromes result from mutations in the $\alpha$ or $\beta$ globulin that disrupts the normal globulin ratio. The presentation of these syndromes is clinically diverse, ranging from a carrier state to a life-threatening hemolytic anemia requiring chronic blood transfusion. Managing patients with transfusiondependent $\beta$-thalassemia major still remains a major challenge to clinicians. Despite an effective transfusion protocol, transfusion hemosiderosis is a major cause of mortality in these patients. However, with a proper, systematic approach from the medical team and regular follow-ups, the complications of iron overload can be detected early and reduced. Proper management requires regular and accurate monitoring of total body iron and timely administration of treatment modalities to reduce iron burden. Fortunately, the emergence of effective iron chelators, although cumbersome to administer, has substantially improved the quality of life and care for these patients.

\section{Disclosure}

The authors declare that there are no conflicts of interest regarding the publication of this paper.

\section{References}

1. Taher AT, Musallam KM, Cappellini MD, Weatherall DJ. Optimal management of $\beta$ thalassaemia intermedia. Br J Haematol. 2011;152(5): $512-523$.

2. Taher AT, Viprakasit V, Musallam KM, Cappellini MD. Treating iron overload in patients with non-transfusion-dependent thalassemia. $\mathrm{Am}$ J Hematol. 2013;88(5):409-415.

3. Ladis V, Berdousi H, Gotsis E, Kattamis A. Deferasirox administration for the treatment of non-transfusional iron overload in patients with thalassaemia intermedia. Br J Haematol. 2010;151(5):504-508.

4. Tony S, Daar S, Elshinawy M, Al-Zadjaly S, Al-Khabori M, Wali Y. T2* MRI in regularly transfused children with thalassemia intermedia: serum ferritin does not reflect liver iron stores. Pediatr Hematol Oncol. 2012;29(6):579-584.

5. Chui DH, Fucharoen S, Chan V. Hemoglobin H disease: not necessarily a benign disorder. Blood. 2003;101(3):791-800.
6. Musallam KM, Rivella S, Vichinsky E, Rachmilewitz EA. Non-transfusion-dependent thalassemias. Haematologica. 2013;98(6):833-844.

7. Qari MH, Wali Y, Albagshi MH, et al. Regional consensus opinion for the management of Beta thalassemia major in the Arabian Gulf area. Orphanet J Rare Dis. 2013;8:143.

8. Northern California Comprehensive Thalassemia Center. Standardof-Care Clinical Practice Guidelines (2012). Available from: http:// thalassemia.com/treatment-guidelines- 4 .aspx $\#$ gsc.tab $=0$. Accessed June 27, 2016.

9. Prabhu R, Prabhu V, Prabhu RS. Iron overload in beta thalassemia: a review. J Biosci Tech. 2009;1(1):20-31.

10. Wood JC. Magnetic resonance imaging measurement of iron overload. Curr Opin Hematol. 2007;14(3):183-190.

11. Wood JC. Diagnosis and management of transfusion iron overload: the role of imaging. Am J Hematol. 2007;82(12 Suppl):1132-1135.

12. Sheth S. SQUID biosusceptometry in the measurement of hepatic iron. Pediatr Radiol. 2003;3(6):373-377.

13. Chapman RW, Williams G, Bydder G, Dick R, Sherlock S, Kreel L. Computed tomography for determining liver iron content in primary haemochromatosis. Br Med J. 1980;280(6212):440-442.

14. Bhat AA, Parwani RN, Wanjari SP. Demonstration of iron in exfoliated buccal cells of $\beta$-thalassemia major patients. J Cytol. 2013;30(3): 169-173.

15. Bacon BR, Britton RS. The pathology of hepatic iron overload: a free radical-mediated process. Hepatology. 1990;11(1):127-137.

16. Engle MA, Erlandson M, Smith CH. Late cardiac complications of chronic, severe, refractory anemia with hemochromatosis. Circulation. 1964;30:698-705.

17. Grundy RG, Woods KA, Savage MO, Evans JP. Relationship of endocrinopathy to iron chelation status in young patients with thalassaemia major. Arch Dis Child. 1994;71(2):128-132.

18. Kwan EY, Lee AC, Li AM, et al. A cross-sectional study of growth, puberty and endocrine function in patients with thalassaemia major in Hong Kong. J Paediatr Child Health. 1995;31(2):83-87.

19. De Sanctis V. Growth and puberty and its management in thalassemia. Horm Res. 2002;58 Suppl 1:72-79.

20. Barry M, Flynn DM, Letsky EA, Risdon RA. Long-term chelation therapy in thalassaemia major: effect on liver iron concentration, liver histology, and clinical progress. Br Med J. 1974;2(5909):16-20.

21. Brittenham GM, Griffith PM, Nienhuis AW, et al. Efficacy of deferoxamine in preventing complications of iron overload in patients with thalassemia major. N Engl J Med. 1994;331(9):567-573.

22. Bronspiegel-Weintrob N, Olivieri NF, Tyler B, Andrews DF, Freedman MH, Holland FJ. Effect of age at the start of iron chelation therapy on gonadal function in $\beta$-thalassemia major. $N$ Engl $J$ Med. 1990;323(11):713-719.

23. Olivieri NF, Nathan DG, McMillan JH, et al. Survival in medically treated patient with homozygous $\beta$-thalassemia. $N$ Engl J Med. 1994;331(9):574-578.

24. Borgna-Pignatti C, Rugolotto S, De Stefano P, et al. Survival and complications in patients with thalassemia major treated with transfusion and deferoxamine. Haemotologica. 2004;89(10):1187-1193.

25. De Virgiliis S, Cossu P, Sanna G, et al. Iron chelation in transfusiondependent thalassemia with chronic hepatitis. Acta Haematol. 1982; 67(1):49-56.

26. Smith RS. Iron excretion in thalassaemia major after administration of chelating agents. Br Med J. 1962;2(5319):1577-1580.

27. Schafer AI, Rabinowe S, Le Boff MS, Bridges K, Cheron RG, Dluhy R. Long-term efficacy of deferoxamine iron chelation therapy in adults with acquired transfusional iron overload. Arch Intern Med. 1985;145(7): 1217-1221.

28. Davis BA, Porter JB. Long-term outcome of continuous 24-hour deferoxamine infusion via indwelling intravenous catheters in high-risk $\beta$-thalassemia. Blood. 2000;95(4):1229-1236.

29. Delea TE, Edelsberg J, Sofrygin O, et al. Consequences and costs of noncompliance with iron chelation therapy in patients with transfusion-dependent thalassemia: a literature review. Transfusion. 2007;47(10):1919-1929. 
30. De Virgillis S, Congia M, Frau F, et al. Deferoxamine-induced growth retardation in patients with thalassemia major. J Pediatr. 1988;113(4): 661-669.

31. Rachmilewitz EA, Giardina PJ. How I treat thalassemia. Blood. 2011;118(13):3479-3488.

32. Cunningham MJ, Macklin EA, Neufield EJ, Cohn AR. Thalassemia Clinical Research Network. Complications of beta-thalassemia major in North America. Blood. 2004;104(1):34-39.

33. Porter JB, Jaswon MS, Huehns ER, East CA, Hazell JW. Desferrioxamine ototoxicity: evaluation of risk factors in thalassaemic patients and guidelines for safe dosage. Br J Haematol. 1989;73(3):403-409.

34. Olivieri NF, Buncic JR, Chew E, et al. Visual and auditory neurotoxicity in patients receiving subcutaneous deferoxamine infusions. $N$ Engl J Med. 1986;314(14):869-873.

35. Yacobovich J, Stark P, Barzilai-Birenbaum S, et al. Acquired proximal renal tubular dysfunction in $\beta$-thalassemia patients treated with deferasirox. J Pediatr Hematol Oncol. 2010;32(7):564-567.

36. Piga A, Roggero S, Salussolia I, Massano D, Serra M, Longo F. Deferiprone. Ann N Y Acad Sci. 2010;1202:75-78.

37. Cappellini MD, Musallam KM, Taher AT. Overview of iron chelation therapy with desferrioxamine and deferiprone. Hemoglobin. 2009;33 Suppl 1:S58-S69.

38. Borgna-Pignatti C, Cappellini MD, De Stefano P, et al. Cardiac morbidity and mortality in deferoxamine-or deferiprone-treated patients with thalassemia major. Blood. 2006;107(9):3733-3737.

39. Mamtani M, Kulkarni H. Influence of iron chelators on myocardial iron and cardiac function in transfusion-dependent thalassaemia: a systematic review and meta-analysis. Br J Haematol. 2008;141(6):882-890.

40. Anderson LJ, Westwood MA, Holden S, et al. Myocardial iron clearance during reversal of siderotic cardiomyopathy with intravenous desferrioxamine: a prospective study using $\mathrm{T} 2 *$ cardiovascular magnetic resonance. Br J Haematol. 2004;127(3):348-355.

41. Hoffbrand AV, Cohen A, Hershko C. Role of deferiprone in chelation therapy for transfusional iron overload. Blood. 2003;102(1):17-24.

42. Cohen AR, Galanello R, Piga A, Dipalma A, Vullo C, Tricta F. Safety profile of the oral iron chelator deferiprone: a multicentre study. $\mathrm{Br} J$ Haematol. 2000;108(2):305-312.

43. Olivieri NF, Brittenham GM, McLaren CE, et al. Long-term safety and effectiveness of iron-chelation therapy with deferiprone for thalassemia major. N Engl J Med. 1998;339(7):417-423.

44. Pippard MJ, Weatherall DJ. Oral iron chelation therapy for thalassaemia: an uncertain scene. Br J Haematol. 2000;111(1):2-5.

45. Tanner MA, Galanello R, Dessi C, et al. A randomized, placebo-controlled, double-blind trial of the effect of combined therapy with deferoxamine and deferiprone on myocardial iron in thalassemia major using cardiovascular magnetic resonance. Circulation. 2007;115(14):1876-1884.
46. Fisher SA, Brunskill SJ, Doree C, Chowdhury O, Gooding S, Roberts DJ. Oral deferiprone for iron chelation in people with thalassaemia. Cochrane Database Syst Rev. 2013;(8):CD004839.

47. Cappellini MD, Cohen A, Piga A, et al. A Phase 3 study of deferasirox (ICL670), a once daily iron chelator, in patients with beta-thalassemia. Blood. 2005;107(9):3455-3462.

48. Pennell DJ, Porter JB, Piga A, et al. A 1-year randomized controlled trial of deferasirox vs deferoxamine for myocardial iron removal in $\beta$-thalassemia major (CORDELIA). Blood. 2014;123(10):1447-1454.

49. Glickstein H, El RB, Shvartsman M, Cabantchik ZI. Intracellular labile iron pools as direct targets of iron chelators: a fluorescence study of chelator action in living cells. Blood. 2005;106(9):3242-3250.

50. Borgna-Pignatti C, Cappellini MD, De Stefano P, et al. Cardiac morbidity and mortality in deferoxamine- or deferiprone-treated patients with thalassemia major. Blood. 2006;107(9):3733-3737.

51. US Food and Drug Administration [homepage on the Internet]. Exjade (deferasirox): Boxed Warning. FDA Safety Alerts for Human Medical Products; 2010. Available from: http://www.fda.gov/Safety/MedWatch/\%20SafetyInformation/SafetyAlertsforHumanMedicalProducts/ ucm200850.htm. Accessed June 28, 2016.

52. Wonke B, Wright C, Hoffbrand AV. Combined therapy with deferiprone and desferrioxamine. Br J Haematol. 1998;103(2):361-364.

53. De Domenico I, Ward DM, Kaplan J. Specific iron chelators determine the route of ferritin degradation. Blood. 2009;114(20):4546-4551.

54. Hoffbrand AV, Taher A, Cappellini MD. How I treat transfusional iron overload. Blood. 2012; 120(18):3657-3669.

55. Olivieri NF, Berriman AM, Tyler BJ, Davis SA, Francombe WH, Liu PP. Continuous intravenous administration of deferoxamine in adults with severe iron overload. Am J Hematol. 1992;41(1):61-63.

56. Ladis V, Chouliaras G, Berdousi H, Kanavakis E, Kattamis C. Longitudinal study of survival and causes of death in patients with thalassemia major in Greece. Ann NY Acad Sci. 2005;1054:445-450.

57. Roudbari M, Soltani-Rad M, Roudbari S. The survival analysis of beta thalassemia major patients in South East of Iran. Saudi Med J. 2008;29(7):1031-1035.

58. Ehlers KH, Giardina PJ, Lesser ML, Engle MA, Hilgartner MW. Prolonged survival in patients with beta-thalassemia major treated with deferoxamine. J Pediatr. 1991;118(4 Pt 1):540-545.

59. Fosburg M, Nathan DG. Treatment of Cooley's anemia. Blood. 1990;76(3):435-444.

60. Loukopoulos D, Voskaridou E, Stamoulakatou A, et al. Hydroxyurea therapy in thalassemia. Ann NY Acad Sci. 1998;850:120-128.

61. Italia KY, Jijina FF, Merchant R, et al. Effect of hydroxyurea on the transfusion requirements in patients with severe HbE-beta-thalassaemia: a genotypic and phenotypic study. J Clin Pathol. 2010;63(2): $147-150$.
Journal of Blood Medicine

\section{Publish your work in this journal}

The Journal of Blood Medicine is an international, peer-reviewed, open access, online journal publishing laboratory, experimental and clinical aspects of all aspect pertaining to blood based medicine including but not limited to: Transfusion Medicine; Blood collection, Donor issues, Transmittable diseases, and Blood banking logistics; Immunohematology; Artificial and alternative blood based therapeutics; Hematology; Biotechnology/nanotechnology of blood related medicine; Legal aspects of blood medicine; Historical perspectives. The manuscript management system is completely online and includes a very quick and fair peer-review system. Visit http://www.dovepress.com/ testimonials.php to read real quotes from published authors. 